\title{
Influence of a temporary stabilization device on respiratory status in patients with severe trauma with a femoral shaft fracture treated by damage control strategy
}

\author{
Camille Maury $^{1} \cdot$ Severin Ramin ${ }^{1} \cdot$ Jordi Bonfils $^{1} \cdot$ Louis Dagneaux $^{2} \cdot$ Patrick Faure $^{2} \cdot$ François Canovas $^{2}$. \\ Xavier Capdevila ${ }^{1} \cdot$ Jonathan $^{\text {Charbit }}{ }^{1}$
}

Received: 25 September 2019 / Accepted: 3 January 2020 / Published online: 17 January 2020

(c) Springer-Verlag GmbH Germany, part of Springer Nature 2020

\begin{abstract}
Background There are few studies on the safety and respiratory consequences of the use of a skeletal traction (ST) device in the management of femoral shaft fractures with damage control orthopaedics (DCO) strategy, particularly in cases of prolonged use. The aim of this study was to assess the influence of ST compared with an external fixator (EF) on respiratory complications and mechanical ventilation requirements in patients with severe trauma with a femoral shaft fracture managed by DCO strategy.

Methods We retrospectively reviewed all patients with severe trauma patients with a unilateral femoral shaft fracture admitted to our institution from 2010 to 2015. Patients who did not undergo definitive osteosynthesis during the first $24 \mathrm{~h}$ were included and divided into two groups: DCO-ST group and DCO-EF group. In addition to trauma severity, global management of respiratory complications, the incidence of acute respiratory distress syndrome (ARDS) and mechanical ventilation requirements and outcome were compared.

Results Fifty-five patients were managed with DCO strategy (mean Injury Severity Score, 28.4); there were 31 in the DCOST group and 24 in the DCO-EF group. No significant difference in terms of the main characteristics, initial severity and associated injuries was observed between the two groups. In contrast, ARDS was found more frequently in the DCO-ST group ( $81 \%$ versus $54 \%$; $P=0.035$ ). Number of ventilation days also tended to be higher in the DCO-ST group (9 days [IQR $3-15$ days] versus 7 [IQR 2-16 days]; $P=0.24$ ). No difference was found for mortality and hospitalization duration between the DCO-ST and DCO-EF groups.

Conclusion The prolonged use of an ST device in the present cohort was associated with a higher incidence of impaired respiratory function. Therefore, our findings suggest that EF is preferable to ST in the DCO setting for femoral shaft fracture, especially in trauma patients at high risk of developing delayed respiratory failure.
\end{abstract}

Keywords Acute respiratory distress syndrome $\cdot$ External fixator $\cdot$ Mechanical ventilation $\cdot$ Multiple organ failure $\cdot$ Skeletal traction

Severin Ramin

severin.ramin@gmail.com

Camille Maury

c-maury@chu-montpellier.fr

Jordi Bonfils

bonfils_8@hotmail.com

Louis Dagneaux

1-dagneaux@chu-montpellier.fr

Patrick Faure

p-faure@chu-montpellier.fr

François Canovas

f-canovas@chu-montpellier.fr
Xavier Capdevila

x-capdevila@chu-montpellier.fr

Jonathan Charbit

j-charbit@chu-montpellier.fr

1 Trauma Intensive and Critical Care Unit, Département D’Anesthésie Réanimation, Lapeyronie University Hospital, 371 Avenue du Doyen G. Giraud, 34295 Montpellier, France

2 Department of Traumatic and Orthopaedic Surgery, Lapeyronie University Hospital, 371 Avenue du Doyen G. Giraud, 34090 Montpellier, France 


\section{Introduction}

Bone injuries occur in more than $75 \%$ of blunt trauma patients in most series [1]. Femoral shaft fractures are regarded as severe injuries because of their association with many complications; significant or massive blood loss, risk of fat embolism, soft tissue damage, and early development of a systemic inflammatory response [2, 3]. Consequently, systemic complications due to inflammatory aggression, acute lung injuries and acute respiratory distress syndrome (ARDS), multiple organ failure (MOF) and a higher mortality are more frequently observed in presence of femoral shaft fractures [4].

Surgical management of femoral shaft fractures has been well described in recent years, leading to significantly improved outcomes in this population [5]. Although many patients benefit from early definitive fixation by intra-medullary nailing, it is nowadays largely agreed that borderline and severe trauma patients have to be managed using damage control orthopaedics (DCO) and sequenced surgeries [5]. In severely injured patients, DCO was associated with reduced inflammation and "second hit" insult compared to early definitive fixation and intra-medullary nailing.

Temporary stabilization of the femoral shaft is usually allowed in a DCO strategy using an external fixator (EF) [6-9]. Some authors have also proposed an alternative device, skeletal traction (ST), described as simple, fast, and less expensive $[10,11]$. The arguments for ST are that its implementation does not require general anaesthesia or transport to the operating room. Nevertheless, few studies have focused on the safety of an ST device in severe or multiple trauma patients, particularly in cases of prolonged use. Delayed fat embolism or respiratory decubitus complications may occur with ST, because this device does not prevent mobilization of the fracture site and imposes a strict dorsal position.

The aim of present study was to assess the influence of ST compared with EF on respiratory complications and mechanical ventilation requirements in severe trauma patients with a unilateral femoral shaft fracture managed by a DCO strategy.

\section{Methods}

\section{Study design and patients}

The hospital charts for the trauma intensive care unit (ICU) of Lapeyronie University Hospital (Level I Regional Trauma Centre, Montpellier, France) were studied retrospectively from January 2010 to December 2015. This unit receives all patients directly from trauma scenes in the area suspected to be severely injured during pre-hospital assessment according to French guidelines [12].

All consecutive patients admitted to this unit with a femoral fracture were screened. Patients with a femoral shaft fracture were included if they were managed by a DCO strategy. Exclusion criteria were as follows: (1) previous admission to another hospital; (2) bilateral shaft femoral fractures; (3) data missing from the medical report; (4) early death within the first $48 \mathrm{~h}$; (5) definitive osteosynthesis within the first $24 \mathrm{~h}$. Because of its retrospective and observational nature, the need for written consent for this study was not required by our institutional ethical committee.

\section{Data collection}

Data on age, sex, mechanism of injuries, and informations according the femoral fracture (Gustilo scale) were extracted from the medical records, as well as information on initial transfusion management, and Glasgow coma scale score, vasopressor use, partial pressure of oxygen in arterial blood/ fraction of inspired oxygen ratio $\left(\mathrm{PaO}_{2} / \mathrm{FiO}_{2}\right.$ ratio) on admission. The Injury Severity Score (ISS), Abbreviated Injury Scale (AIS) by body region, Thoracic Trauma Severity (TTS) score, a Sepsis-related Organ Failure Assessment (SOFA) scores were calculated for each patient [13-16]. Details on femoral surgical management during hospitalization (timing and type of initial and definitive fixation) were also collected. Finally, some outcome data were recorded: (1) orthopaedic outcome (mal-union, non-union and local sepsis); (2) respiratory complications (mechanical ventilation duration, extubation failure rate, $\mathrm{PaO}_{2} / \mathrm{FiO}_{2}$ ratio between day 1 and day 15 (obtained daily at $8 \mathrm{am}$ ), ARDS during the first 15 days of hospitalization; (3) general outcome (severe bedsores, transfusion requirements, vasopressor and sedation duration, daily SOFA scores, ICU and hospital length of stay, MOF and mortality).

\section{DCO management}

DCO strategy was applied in our institution following the usual recommendations (i.e., traumatic brain injury, haemorrhagic or hypoxemic injuries to the trunk, severe coagulopathy, circulatory instability, acute organ failure, etc.) [5]. During the study period, our trauma centre experienced a significant shift in practice concerning the device used for temporary fixation. This change was introduced according to an institutional protocol from 2014. If ST was initially placed in a DCO setting, EF was increasingly used following this protocol. ST was thus progressively abandoned. Choice of the DCO device, whether EF or ST, was decided by the attending surgeon. Definitive stabilization 
was subsequently achieved when the clinical status of patient allowed placement of femoral intra-medullary nailing. In parallel, Patients with chest trauma were managed according a multi-disciplinary approach including respiratory management, surgical stabilization and effective physiotherapy. All intubated patients were mobilized every two hours. Backrest position with $45^{\circ}$ angle was achieved as preconized if possible. Lateral recumbency was also used in case of backrest position was prohibited.

\section{Study definitions}

Following an initial analysis, two groups were defined according to the kind of DCO used during the first $24 \mathrm{~h}$ : the DCO-ST group included patients who benefited from ST for temporary stabilization; the DCO-EF group included patients who benefited from an external fixator.

Mechanical ventilation was considered if the patient was ventilated for least $24 \mathrm{~h}$, excluding mechanical ventilation administrated in the operating room. Weaning failure was defined by the need to re-intubate within $24 \mathrm{~h}$ of extubation.

The main outcome criterion was the occurrence of ARDS during the 15 first days after admission, according to the international Berlin definition [17]. This definition implies the presence of bilateral opacity on chest imaging and hypoxia not fully explained by cardiac failure or fluid overload. ARDS was staged as mild $\left(\mathrm{PaO}_{2} / \mathrm{FiO}_{2}\right.$ between 300 and 201), moderate $\left(\mathrm{PaO}_{2} / \mathrm{FiO}_{2}\right.$ between 101 and 200) and severe $\left(\mathrm{PaO}_{2} / \mathrm{FiO}_{2} \leq 100\right)$. If the patient was not intubated, $\mathrm{FiO}_{2}$ was determined using the rules described by Wagstaff et al. [16] to calculate the $\mathrm{PaO}_{2} / \mathrm{FiO}_{2}$ ratio.
MOF was defined by a SOFA score $\geq 10$. As in many series, SOFA was calculated without neurological status because of the wide use of sedation in this population.

\section{Statistical analysis}

The main demographic data of the patients and characteristics on admission and during hospitalization were assessed according to the DCO strategy using bivariate analysis: DCO-ST group and DCO-EF group. Respiratory status, mechanical ventilation requirements and ARDS were specifically analysed. Continuous data were expressed as means (standard deviation [SD]) when parametric, or medians [interquartile range (IQR)] when non-parametric. Comparisons between these groups were performed using the Student $t$ test for continuous parametric data and the Mann-Whitney $U$ test for non-parametric data. Categorical data were expressed as number (percentage) and compared using Chi square or Fisher's exact tests as appropriate. Statistical analysis was performed using XLSTAT Pro 5.7.2 (Addinsoft, New York, NY). $P \leq 0.05$ indicated significance.

\section{Results}

\section{Patient characteristics}

During the 5-year study period, 272 severe trauma patients were admitted to our trauma centre with at least one femoral fracture. Of these, 112 were excluded from the analysis; 27 because of indirect or delayed admissions, 3 early deaths, 57 non-shaft fractures, 22 bilateral femoral shaft fractures and 3 with a lack of clinical data (Fig. 1). Of the

Fig. 1 Flow chart

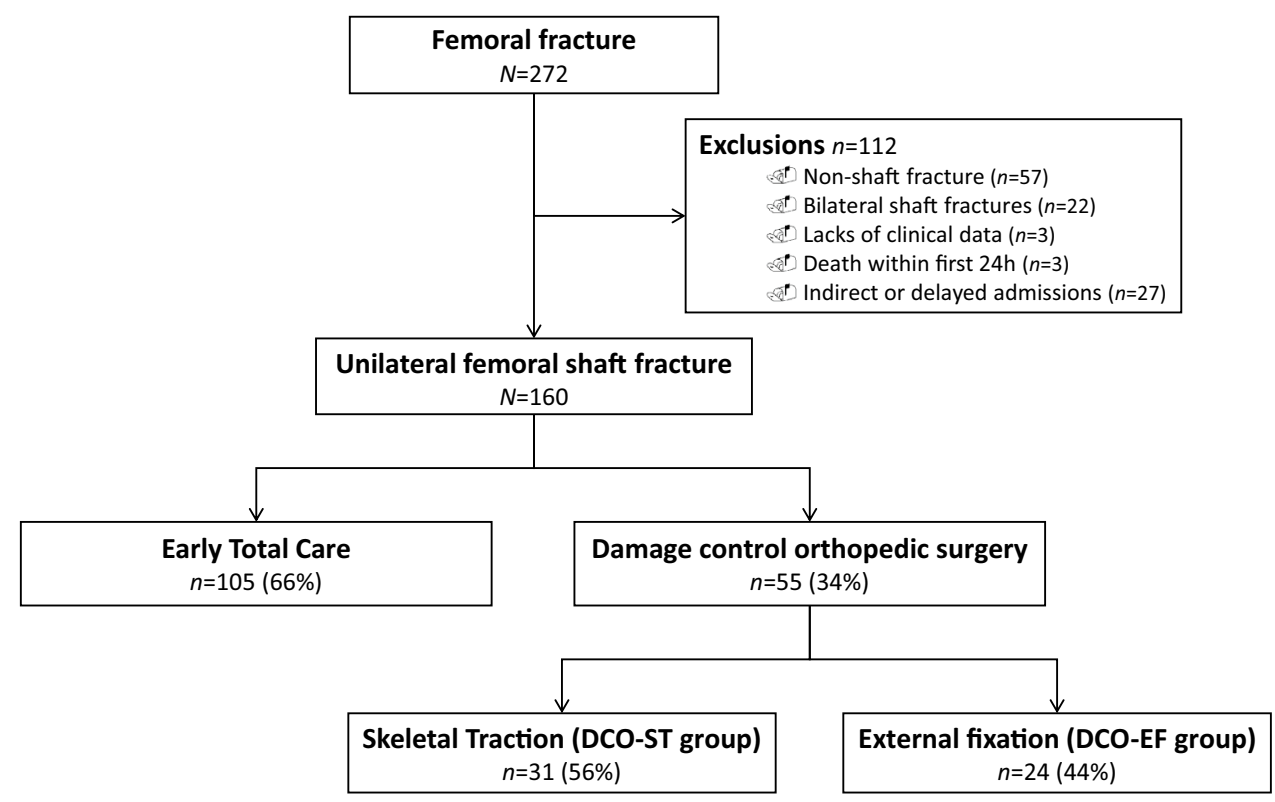


Fig. 2 Surgical management of unilateral femoral shaft fracture
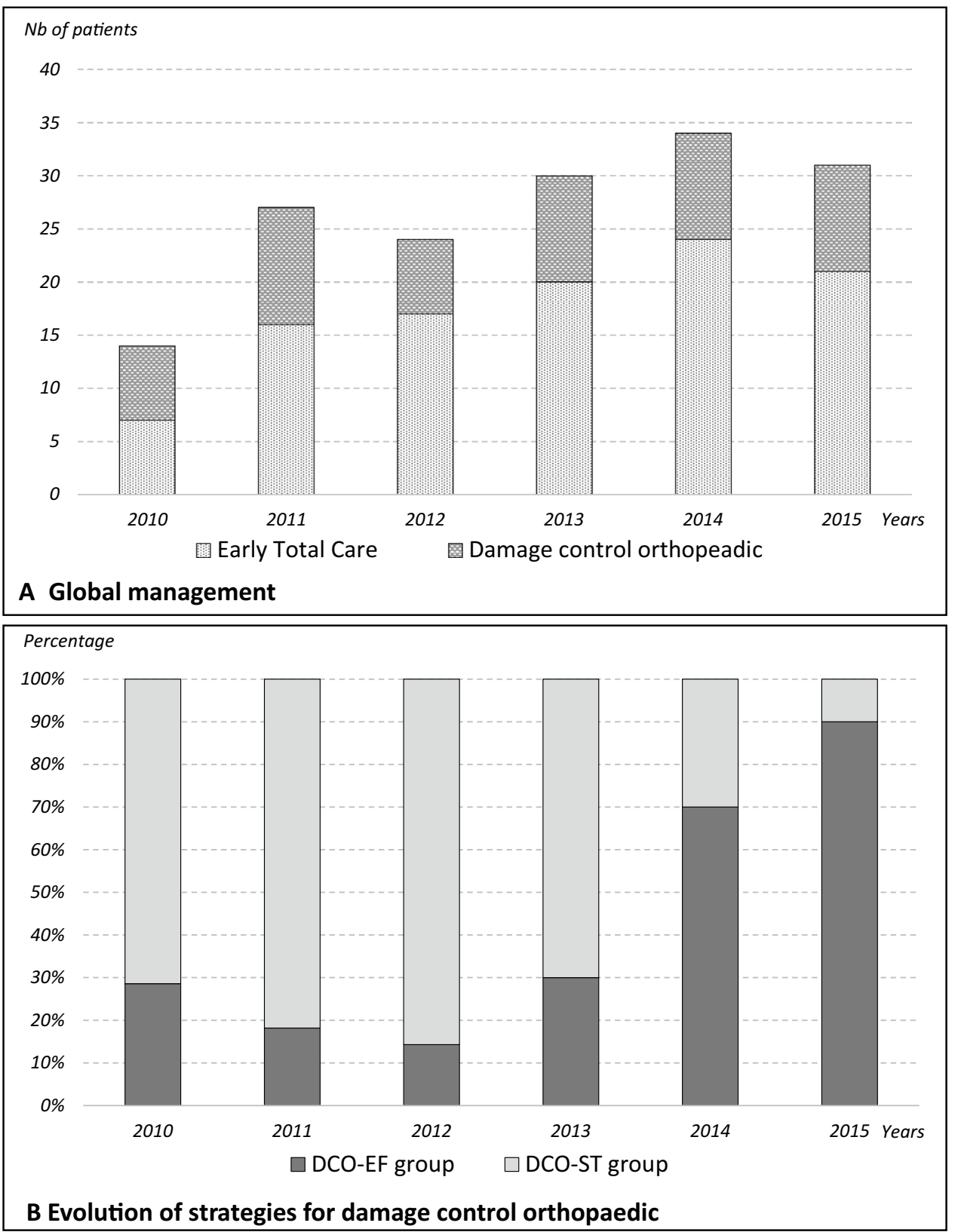

160 remaining patients, 105 (66\%) benefited from initial definitive osteosynthesis and 55 (34\%) underwent DCO management (Fig. 2).

In the DCO population, 47 patients $(85 \%)$ were male, the mean age was 32.6 years (SD 17.3 years), the mean ISS was 27 (IQR 19-34) and 28 patients (51\%) had a head AIS $\geq 3$. Motor vehicle and bicycle accidents were the main mechanisms of injury. The characteristics of these patients are summarized in Table 1. A total of 47 (85\%) patients received mechanical ventilation during hospitalization, with a mean duration of 10.9 days (SD 7.5 days).

\section{Damage control orthopaedic groups}

Among the cohort, 24 patients (44\%) were temporary stabilized by EF (DCO-EF group) and $31(56 \%)$ by ST (DCO-ST group). Table 1 shows the absence of a significant difference between the two groups regarding the main severity parameters on admission (severity criteria or transfusion needs) or respiratory severity (mechanical ventilation rate, $\mathrm{PaO}_{2} / \mathrm{FiO}_{2}$ or TTS score). The rate of open fractures was $29 \%$ in the DCO-EF group versus $19 \%$ in the 
Table 1 Characteristics of patients treat by damage control orthopaedic surgery

\begin{tabular}{|c|c|c|c|c|c|c|c|}
\hline \multirow[b]{2}{*}{ No patients } & \multicolumn{2}{|l|}{ All patients } & \multicolumn{2}{|l|}{ DCO-EF group } & \multicolumn{2}{|l|}{ DCO-ST group } & \multirow[t]{2}{*}{$P$ value } \\
\hline & \multicolumn{2}{|l|}{$N=55$} & \multicolumn{2}{|l|}{$n=24$} & \multicolumn{2}{|l|}{$n=31$} & \\
\hline \multicolumn{8}{|l|}{ Main characteristics } \\
\hline Age (Years) & \multicolumn{2}{|l|}{$28.0[21.3-44.0]$} & \multicolumn{2}{|l|}{$27.5[19.0-47.4]$} & \multicolumn{2}{|l|}{$33.0[22.0-44.0]$} & $0.70 \dagger$ \\
\hline Age $\geq 55$ years, $n(\%)$ & \multicolumn{2}{|l|}{$8(15 \%)$} & \multicolumn{2}{|l|}{$3(13 \%)$} & \multicolumn{2}{|l|}{$5(16 \%)$} & $0.99 \ddagger$ \\
\hline Male, $n(\%)$ & \multicolumn{2}{|l|}{$47(85 \%)$} & $20(83 \%)$ & & \multicolumn{2}{|l|}{$27(87 \%)$} & $0.99 \ddagger$ \\
\hline \multicolumn{8}{|l|}{ Mechanism of trauma, $n(\%)$} \\
\hline Motor vehicle crash & \multicolumn{2}{|l|}{$35(64 \%)$} & $16(67 \%)$ & & \multicolumn{2}{|l|}{$19(61 \%)$} & $0.32 \ddagger$ \\
\hline Bicycle & \multicolumn{2}{|l|}{$11(20 \%)$} & $6(25 \%)$ & & \multicolumn{2}{|l|}{$5(16 \%)$} & \\
\hline Pedestrian & $3(5 \%)$ & & $1(4 \%)$ & & $2(6 \%)$ & & \\
\hline Fall & $3(5 \%)$ & & $1(4 \%)$ & & $2(6 \%)$ & & \\
\hline Assault & $3(5 \%)$ & & $0(0 \%)$ & & $3(10 \%)$ & & \\
\hline Injury severity & & & & & & & \\
\hline ISS & 27 [19-34] & & $26[17-34]$ & & $27[22-36]$ & & $0.43 \dagger$ \\
\hline ISS $\geq 16, n(\%)$ & $48(87 \%)$ & & $21(88 \%)$ & & $27(87 \%)$ & & $0.72 \ddagger$ \\
\hline Severe anatomical injuries, $n(\%)$ & & & & & & & \\
\hline Any AIS $\geq 3$ & $50(91 \%)$ & & $22(92 \%)$ & & $28(90 \%)$ & & $0.62 \ddagger$ \\
\hline Head AIS $\geq 3$ & $28(51 \%)$ & & $10(42 \%)$ & & $18(58 \%)$ & & $0.23 \ddagger$ \\
\hline Face AIS $\geq 3$ & $8(15 \%)$ & & $3(13 \%)$ & & $5(16 \%)$ & & $0.99 \ddagger$ \\
\hline Chest AIS $\geq 3$ & $17(31 \%)$ & & $10(42 \%)$ & & $7(23 \%)$ & & $0.13 \ddagger$ \\
\hline Abdominal $\geq 3$ & $13(24 \%)$ & & $5(21 \%)$ & & $8(26 \%)$ & & $0.67 \ddagger$ \\
\hline Bones* AIS $\geq 3$ & $48(87 \%)$ & & $22(92 \%)$ & & $26(84 \%)$ & & $0.65 末$ \\
\hline Open femoral shaft fracture, $n(\%)$ & & & & & & & \\
\hline Overall open fracture & $13(24 \%)$ & & $7(29 \%)$ & & $6(19 \%)$ & & $0.40 \$$ \\
\hline Gustilo 1 & $3(5 \%)$ & & $2(8 \%)$ & & $1(3 \%)$ & & \\
\hline Gustilo 2 & $7(13 \%)$ & & $3(13 \%)$ & & $4(13 \%)$ & & \\
\hline Gustilo 3 & 3 & $(5 \%)$ & 2 & $(8 \%)$ & 1 & $(3 \%)$ & \\
\hline GCS score on arrival & $10.0[6.0-15.0]$ & & $10.5[6.0-15.0]$ & & $9.0[5.0-15.0]$ & & $0.76 \dagger$ \\
\hline Admission vasopressor use, $n(\%)$ & $45(82 \%)$ & & $19(79 \%)$ & & $26(84 \%)$ & & $0.92 \ddagger$ \\
\hline Admission SOFA score & $2.0[0.0-5.0]$ & & $3.0[1.0-6.0]$ & & $2.0[0.0-4.0]$ & & $0.11 \dagger$ \\
\hline TRISS score & $2.6[1.0-3.5]$ & & $3.1[1.6-3.8]$ & & $2.4[1.0-3.0]$ & & $0.10 \dagger$ \\
\hline Admission SAPS II score & $34[26-43]$ & & $33[25-43]$ & & $34[27-44]$ & & $0.92 \dagger$ \\
\hline Respiratory severity criteria & & & & & & & \\
\hline TTS score & $5[2-8]$ & & $5[3-9]$ & & $4[2-8]$ & & $0.75 \dagger$ \\
\hline Number of ribs fracture & $3[0-7]$ & & $3[2-9]$ & & $3[0-7]$ & & $0.35 \dagger$ \\
\hline Flail chest (\%) & $9(16 \%)$ & & $4(17 \%)$ & & $5(16 \%)$ & & $0.67 \ddagger$ \\
\hline Lung contusion $(\%)$ & $18(33 \%)$ & & $9(38 \%)$ & & $9(29 \%)$ & & $0.51 \ddagger$ \\
\hline Admission $\mathrm{PaFi}$ & $330[232-407]$ & & $334[231-436]$ & & $323[232-380]$ & & $0.54 \dagger$ \\
\hline Prehospital mechanical ventilation, $n(\%)$ & $34(62 \%)$ & & $13(54 \%)$ & & $21(68 \%)$ & & $0.30 \div$ \\
\hline Mechanical ventilation in first $24 \mathrm{~h}, n(\%)$ & $43(78 \%)$ & & $18(75 \%)$ & & $25(81 \%)$ & & $0.62 \div$ \\
\hline Initial transfusion management & & & & & & & \\
\hline Requirements in the first $24 \mathrm{~h}$ & & & & & & & \\
\hline Number of RBC (Units) & $4.0[0.0-6.0]$ & & $4.0[1.0-8.5]$ & & $3.0[0.0-5.0]$ & & $0.24 \dagger$ \\
\hline Number of FFP (Units) & $2.0[0.0-6.0]$ & & $3.0[0.0-6.5]$ & & $2.0[0.0-5.0]$ & & $0.40 \dagger$ \\
\hline Number of platelets (Units) & $0.0[0.0-1.0]$ & & $0.0[0.0-1.0]$ & & $0.0[0.0-0.0]$ & & $0.03 \dagger$ \\
\hline Massive transfusion, $n(\%)$ & $9(16 \%)$ & & $6(25 \%)$ & & $3(10 \%)$ & & $0.13 \ddagger$ \\
\hline
\end{tabular}

Data are expressed as median [IQR] or as number of patients (percentage) as appropriate

* Not including femoral fracture

$\dagger$ Mann-Whitney test

$¥$ Chi-square or Fisher test as appropriate

ISS injury severity score, AIS Abbreviated Injury Scale, GCS score Glasgow coma scale score, SOFA sepsis-related organ failure assessment, TRISS score Trauma Related Injury Severity Score, SAPS II simplified acute physiology score, $\mathrm{PaFi} \mathrm{PaO}_{2} / \mathrm{FiO} \mathrm{O}_{2}$ ratio, TTS score, Thoracic trauma severity score, $R B C$ red blood cells, $F F P$ fresh frozen plasma 
Table 1 (continued)

Massive transfusion: $\geq 10$ CGR during first $24 \mathrm{~h}$

Table 2 Surgical management during hospitalization

\begin{tabular}{lllll}
\hline & All patients & DCO-EF group & DCO-ST group & $P$ value \\
\hline $\begin{array}{l}\text { No patients } \\
\text { Late femoral management }\end{array}$ & $N=55$ & $n=24$ & $n=31$ & \\
$\quad$ Time to definitive fixation (Hours) & $117[59-256]$ & $161[96-267]$ & $95[39-220]$ & $0.046^{\dagger}$ \\
Definitive fixation, $n$ (\%) & & & \\
$\quad$ External Fixator & $6(11 \%)$ & $6(25 \%)$ & $0(0 \%)$ & $0.004^{\ddagger}$ \\
$\quad$ Intramedullary nailing & $49(89 \%)$ & $18(75 \%)$ & $31(100 \%)$ & \\
Extrafemoral surgical management, $n(\%)$ & & & \\
Any intervention & $41(73 \%)$ & $18(71 \%)$ & $23(74 \%)$ & $0.95^{\ddagger}$ \\
Orthopaedic surgery & $36(65 \%)$ & $17(71 \%)$ & $19(61 \%)$ & $0.46^{\ddagger}$ \\
Neurosurgery & $5(9 \%)$ & $2(8 \%)$ & $3(10 \%)$ & $0.39^{\ddagger}$ \\
Laparotomy & $6(11 \%)$ & $4(17 \%)$ & $2(6 \%)$ & $0.65^{\ddagger}$ \\
Thoracotomy & $2(4 \%)$ & $1(4 \%)$ & $1(3 \%)$ & $1.00^{\ddagger}$ \\
$\quad$ Vascular surgery & $3(5 \%)$ & $2(8 \%)$ & $1(3 \%)$ & $0.57^{\ddagger}$ \\
Maxillofacial surgery & $5(9 \%)$ & $3(13 \%)$ & $2(6 \%)$ & $0.64^{\ddagger}$ \\
Trans-arterial embolization & $1(2 \%)$ & $1(4 \%)$ & $0(0 \%)$ & $0.43^{\ddagger}$ \\
Other & $3(5 \%)$ & $2(8 \%)$ & $1(3 \%)$ & $0.57^{\ddagger}$ \\
Mean of extrafemoral intervention & $1[0-2]$ & $1[0-2]$ & $1[0-2]$ & $0.97^{\dagger}$ \\
\hline
\end{tabular}

Data are expressed as median [IQR] or as number of patients (percentage) as appropriate

${ }^{\dagger}$ Mann-Whitney test

${ }^{\ddagger}$ Chi-square or Fisher test as appropriate
DCO-ST group $(P=0.40)$, and the rate of massive transfusion was $25 \%$ versus $10 \%$, respectively $(P=0.13)$.

\section{Surgical management}

Definitive femoral management and details of other surgical management are summarized in Table 2 . The delay in definitive shaft fixation was significantly longer for the DCO-EF group versus the DCO-ST group: $161 \mathrm{~h}$ [IQR 96-267 h] versus $95 \mathrm{~h}$ [IQR $39-220 \mathrm{~h}$ ] $(P=0.046)$. For 6 patients in the DCO-EF group (25\%), EF was the definitive treatment of the femoral fracture.

During the hospital stay, 61 extra-femoral interventions were achieved in 41 patients (Table 2). The median rate of extra-femoral interventions was similar in the two groups: 1.0 [IQR 0-2] versus 1.0 [IQR 0-2], respectively $(P=0.97)$.

\section{Local complications}

Nine patients (16\%) in this cohort experienced at least one local complication: non-union, mal-union or local sepsis. The overall rate of femoral re-operation was $12 \%$ in the DCO-EF group versus 19\% in the DCO-ST group $(P=0.75$;
Table 3). No difference was observed concerning the kind of local complication.

\section{Respiratory status and mechanical ventilation}

Mechanical ventilation requirements during hospitalization was $92 \%$ in the DCO-EF group versus $81 \%$ in the DCO-ST group $(P=0.84)$. The daily mechanical ventilation requirements for each group are presented in Fig. $3 \mathrm{a}$. Duration of ventilation tended to be shorter in the DCOEF group than in the DCO-ST group (6.75 days [IQR 1.75-16 days] versus 8.5 days [IQR 3.0-14.75 days]) but without a significant difference $(P=0.24)$ (Table 3$)$. During the first 2 weeks, ARDS was less frequent in the DCO-EF group than in the DCO-ST group: all ARDS, $54 \%$ versus $81 \%(P=0.035)$; moderate or severe ARDS, $29 \%$ versus $48 \%(P=0.14)$ (Fig. $4 \mathrm{a}$; Table 3$)$. Median time for onset of ARDS was comparable between the two groups: 1.0 days [IQR $0-2.5$ days] versus 1.5 days [IQR $0-3.0$ days] $(P=0.76)$. The median duration of ARDS was 2.5 days [IQR 1.0-5.0 days] versus 3.5 days [IQR 1.0-6.5 days] $(P=0.17)$. Median worst $\mathrm{PaO}_{2} / \mathrm{FiO}_{2}$ ratio was $260 \mathrm{mmHg}$ [IQR $160-342 \mathrm{mmHg}$ in the DCO-EF group versus $206 \mathrm{mmHg}$ [IQR $152-281 \mathrm{mmHg}$ ] in the DCO-ST group $(P=0.21)$ (Table 3$)$. 
Table 3 Complications and outcomes

\begin{tabular}{|c|c|c|c|c|}
\hline & All patients & DCO-EF group & DCO-ST group & $P$ value \\
\hline No patients & $N=55$ & $n=24$ & $n=31$ & \\
\hline \multicolumn{5}{|l|}{ Local complications, $n(\%)$} \\
\hline Overall local complications & $9(16 \%)$ & $3(12 \%)$ & $6(19 \%)$ & $0.75^{*}$ \\
\hline Non-union & $5(9 \%)$ & $1(4 \%)$ & $4(13 \%)$ & $0.37 *$ \\
\hline Mal-union & $1(2 \%)$ & $0(0 \%)$ & $1(3 \%)$ & $1.00 *$ \\
\hline Local sepsis & $3(5 \%)$ & $2(8 \%)$ & $1(3 \%)$ & $0.57 *$ \\
\hline \multicolumn{5}{|l|}{ Respiratory evolution } \\
\hline \multicolumn{5}{|l|}{ Respi SOFA } \\
\hline Admission & $0.0[0.0-1.0]$ & $0.5[0.0-1.5]$ & $0.0[0.0-1.0]$ & $0.15^{\dagger}$ \\
\hline $\mathrm{J} 1$ & $1.0[0.0-1.5]$ & $0.5[0.0-1.0]$ & $1.0[0.0-2.0]$ & $0.13^{\dagger}$ \\
\hline $\mathrm{J} 2$ & $1.0[0.0-2.0]$ & $1.0[0.0-2.0]$ & $1.0[0.0-2.0]$ & $0.25^{\dagger}$ \\
\hline $\mathrm{J} 3$ & $1.0[0.0-1.0]$ & $0.0[0.0-1.0]$ & $1.0[0.0-2.0]$ & $0.06^{\dagger}$ \\
\hline $\mathrm{J} 7$ & $1.0[0.0-2.0]$ & $1.0[0.0-2.0]$ & $1.0[0.0-2.0]$ & $0.57^{\dagger}$ \\
\hline $\mathrm{J} 10$ & $1.0[0.0-2.0]$ & $1.0[0.0-2.0]$ & $1.0[0.0-2.0]$ & $0.91^{\dagger}$ \\
\hline $\mathrm{J} 15$ & $0.0[0.0-2.0]$ & $0.0[0.0-2.0]$ & $0.0[0.0-2.0]$ & $0.76^{\dagger}$ \\
\hline $\mathrm{J} 30$ & $0.0[0.0-0.0]$ & $0.0[0.0-0.0]$ & $0.0[0.0-0.0]$ & $0.88^{\dagger}$ \\
\hline Worst PaFi in first 10 days & $221[157-301]$ & $260[160-342]$ & $206[152-281]$ & $0.21^{\dagger}$ \\
\hline MV requirements, $n(\%)$ & $47(85 \%)$ & $22(92 \%)$ & $25(81 \%)$ & $0.84 *$ \\
\hline MV duration (Days) & $8[3-15]$ & $7[2-16]$ & $9[3-15]$ & $0.24^{\dagger}$ \\
\hline Extubation failure, $n(\%)$ & $3(5 \%)$ & $1(4 \%)$ & $2(6 \%)$ & $0.60^{*}$ \\
\hline \multicolumn{5}{|l|}{ ARDS occurrence, $n(\%)$} \\
\hline All ARDS & $38(69 \%)$ & $13(54 \%)$ & $25(81 \%)$ & $0.035^{*}$ \\
\hline Moderate and severe ARDS & $22(40 \%)$ & $7(29 \%)$ & $15(48 \%)$ & $0.14^{*}$ \\
\hline Pneumonia, $n(\%)$ & $14(25 \%)$ & $5(21 \%)$ & $9(29 \%)$ & $0.53 *$ \\
\hline \multicolumn{5}{|l|}{ General outcome } \\
\hline Severe bedsores, $n(\%)$ & $2(4 \%)$ & $1(4 \%)$ & $1(3 \%)$ & $0.69 *$ \\
\hline \multicolumn{5}{|l|}{ Hospitalization transfusion requirements } \\
\hline Number of RBC (units) & $7.0[2.5-13.0]$ & $8.5[3.5-17.5]$ & $6.0[2.0-11.0]$ & $0.21^{\dagger}$ \\
\hline Number of FFP (units) & $3.0[0.0-6.5]$ & $3.5[0.0-7.5]$ & $3.0[0.0-5.0]$ & $0.46^{\dagger}$ \\
\hline Number of platelets (units) & $0.0[0.0-0.5]$ & $0.0[0.0-1.5]$ & $0.0[0.0-0.0]$ & $0.09^{\dagger}$ \\
\hline Sedation duration $(\mathrm{h})$ & $142[54-221]$ & 96 [20-192] & $160[72-240]$ & $0.21^{\dagger}$ \\
\hline Catecholamines duration (days) & $2.75[1.0-7.75]$ & $2.5[0.6-8.5]$ & $2.75[1.25-7.25]$ & $0.91^{\dagger}$ \\
\hline Acute kidney injury (stage 1 or more) & $15(27 \%)$ & $7(29 \%)$ & $8(26 \%)$ & $0.78^{*}$ \\
\hline Renal replacement therapy, $n(\%)$ & $5(9 \%)$ & $3(13 \%)$ & $2(6 \%)$ & $0.38^{*}$ \\
\hline LOS in ICU (Days) & $13[8-22]$ & $12[7-23]$ & $13[10-20]$ & $0.54^{\dagger}$ \\
\hline LOS in hospital (Days) & $28[21-36]$ & 30 [19-45] & $24[21-34]$ & $0.75^{\dagger}$ \\
\hline MOF, $n(\%)$ & $13(24 \%)$ & $6(25 \%)$ & $7(23 \%)$ & $0.83^{*}$ \\
\hline Hospital mortality, $n(\%)$ & $7(13 \%)$ & $3(13 \%)$ & $4(13 \%)$ & $0.96^{*}$ \\
\hline
\end{tabular}

Data are expressed as median [IQR] or as number of patients (percentage) as appropriate

SOFA sepsis-related organ failure assessment, $\mathrm{PaFi} \mathrm{PaO}_{2} / \mathrm{FiO}_{2}$ ratio, $M V$ mechanical ventilation, ARDS acute respiratory distress syndrome, $R B C$ red blood cells, $F F P$ fresh frozen plasma, $L O S$ length of stay, $I C U$ intensive care unit, $M O F$ multi organ failure (SOFA score $\geq 10$ )

*Chi-square or Fisher test as appropriate

${ }^{\dagger}$ Mann-Whitney test

\section{General complications and outcomes}

Median duration of sedation tended to be shorter in the DCO-EF group than in the DCO-ST group: $92 \mathrm{~h}$ [IQR 20-192 h] versus $160 \mathrm{~h}$ [IQR $72-240 \mathrm{~h}](P=0.21)$.
Occurrence of MOF in the first 2 weeks was comparable between the two groups ( 25 versus 23 , respectively; $P=0.83$ ) (Table 3). Median duration of use of catecholamines was also comparable ( 2.5 days [IQR 0.6-8.5 days] versus 2.75 days [IQR $1.25-7.25$ days]; $P=0.91$ ). Similarly, 
Fig. 3 Comparison of respiratory status between DCO-EF and DCO-ST groups
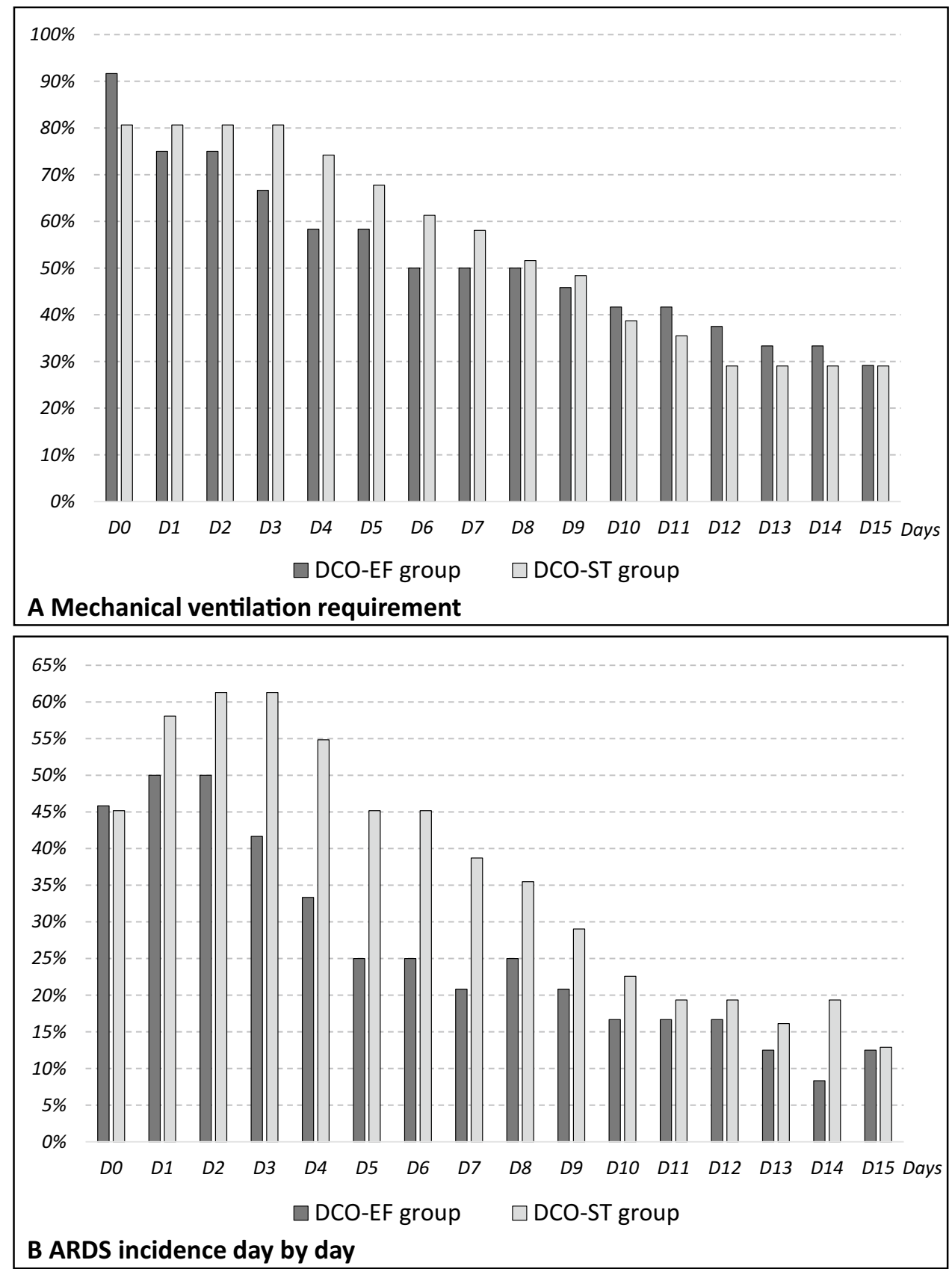

no significant difference was found regarding hospitalization duration and mortality between the two groups (Table 3).

\section{Discussion}

In the present study, we report on a cohort of 55 consecutive patients with multiple trauma including a unilateral femoral shaft fracture and managed by a DCO strategy. The ventilation rate in the overall population was high (85) and trauma severity was also high (mean ISS 27). Median delay of definitive femoral osteosynthesis was $117 \mathrm{~h}$ [IQR
59-256 h] and median duration of mechanical ventilation was 8.0 days [IQR 3.0-15.5 days]. Of this population, 24 (44) were allocated to the DCO-EF group and 31 (56) to the DCO-ST group. Among the main findings, ARDS incidence was higher in the DCO-ST group despite comparable initial severity ( 81 versus $54 ; P=0.035$ ). Duration of mechanical ventilation tended to be higher in the DCO-ST group (8.5 versus 6.75 days; $P=0.24)$. Conversely, no statistical difference was found for hospitalization duration, MOF incidence or mortality.

For many years, early total care was the standard of care for most femoral shaft fractures. The main goal of 
Fig. 4 Respiratory and general outcomes
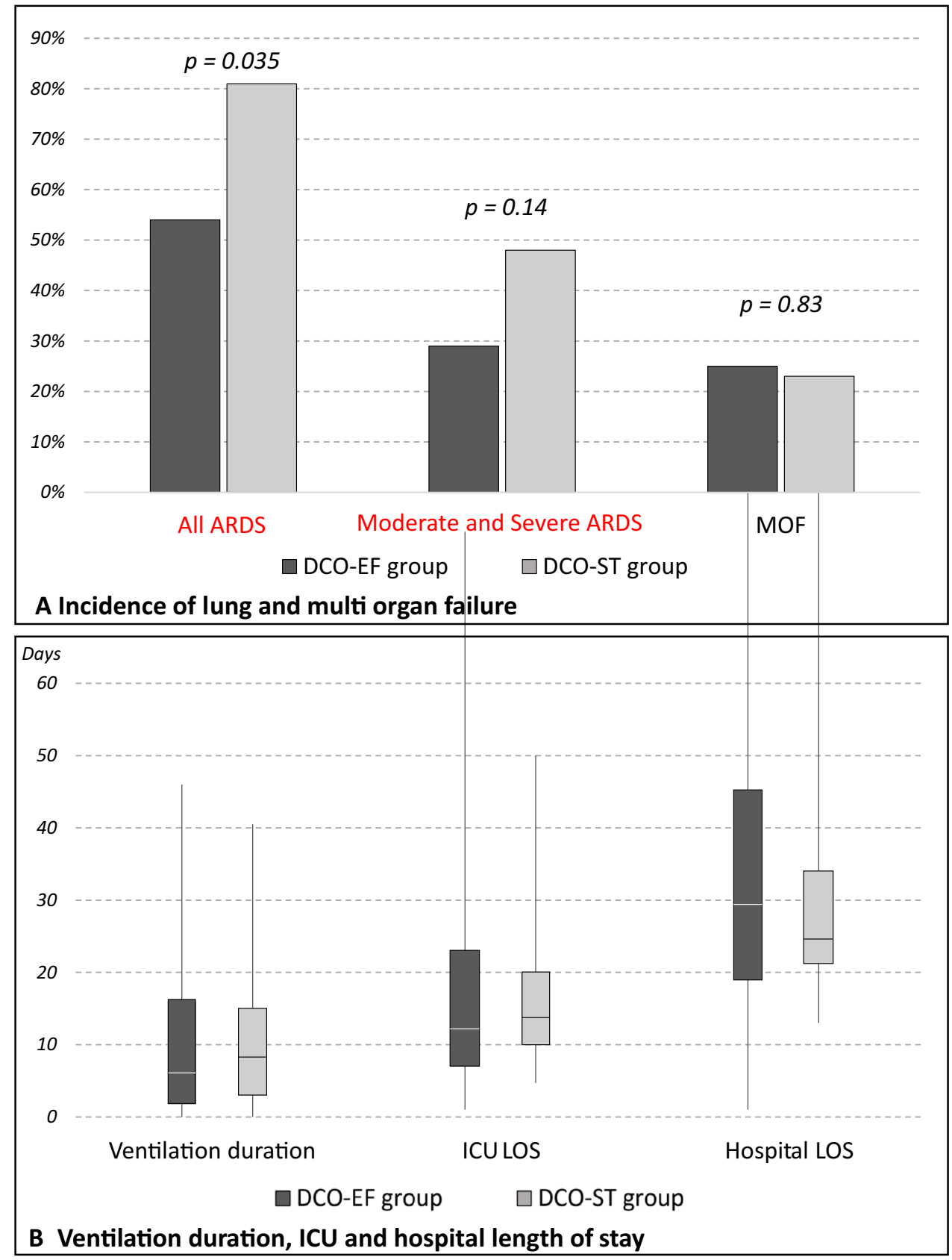

this surgical strategy was to fix the fracture site rapidly and reduce respiratory complications generated by a fat embolism [18, 19]. The development of the "inflammatory concept" in the late 1990s, introduced the principle of operative burden caused by aggressive and early surgical interventions, especially intra-medullary nailing [20, 21]. This second aggression following severe trauma, also called "second hit", was indeed incriminated in the generation of a post-operative inflammatory response responsible for clinical systemic disorders, ARDS and MOF [20-22].

Consequently, the DCO strategy was developed and is now recommended by most experts for borderline and severe trauma patients with long bone fractures [5, 6, 23-25]. In these patients, DCO has proved to be time saving, effective and safe [8]. DCO permits effective temporary stabilization while focusing on resuscitation and restoration of homeostasis in critical patients [5, 6, 23-26]. DCO management is associated with a decrease in the systemic inflammatory insult that results from early orthopaedic surgery and intramedullary hyperpressure [27].

All the evidences on the benefits of DCO were reported using EF for temporary stabilization. EF is a device that allows fixation of the fracture site, maintaining the anatomic axis of broken bones. On the other hand, ST was proposed as 
an alternative to EF because of its simplicity and the speed with which it can be applied. However, only one series has described the safety and efficiency of ST in DCO management. Scannel et al. [11] thus reported a retrospective study comparing EF versus ST in terms of pulmonary complications, MOF and mortality. These authors did not highlight statistical differences for the main endpoints and, therefore, concluded that ST could be a valuable and safe alternative to EF. However, these conclusions must be regarded with caution because of important limitations in this study. First, it was monocentric, retrospective study with a small sample. Second, the definition of ARDS in this study was questionable (i.e., $\mathrm{PaO}_{2} / \mathrm{FIO}_{2} \leq 200 \mathrm{mmHg}$ for 5 consecutive days or more), which explains the low incidence of ARDS of around 5\%. The incidence of ARDS in DCO management is usually around $15-30$ in the literature $[5,6,25,26]$. This methodological bias probably led to a significant lack of statistical power and an erratic prevalence of ARDS. Finally, severity of respiratory criteria probably does not allow objective assessment of all respiratory changes. The safety of ST use in patients at high risk of respiratory failure must consequently still be demonstrated, especially when its use is prolonged. Many constraints have been suggested in the case of ST implantation. First, continuous fixation of the fracture site is not ensured, which may promote delayed fat embolism, supplementary soft injuries and higher blood loss, mal-union and non-union. Second, the traction device strongly limits mobilization of the patient because of unreliable continuous stabilization and risk of micro-displacement at the fracture site. Therefore, it is reasonable to wonder about potential risks of respiratory impairments induced by ST in patients with severe trauma.

In our series of severe trauma patients (mean ISS 27), where $91 \%$ had at least one extra-femoral severe injury and 85 required mechanical ventilation, the ARDS incidence was significantly higher in the DCO-ST group than in the DCO-EF group ( $81 \%$ versus 54\%; $P=0.035$ ). This difference would seem to be maximal in the first week (Fig. 3). Moreover, the duration of ventilation tended to be longer in the DCO-ST group (8.5 days [IQR 3.0-14.75 days] versus 6.75 days [IQR $1.75-16$ days]; $P=0.24$ ). This comparison probably lacks statistical power to highlight a significant difference. The worse outcome was thus observed in the DCOST group even though the two groups were comparable in terms of initial respiratory status and trauma severity on admission (Table 1). Our data, therefore, support a potential adverse risk to respiratory function with prolonged use of an ST device. One explanation could be that ST requires a permanent dorsal decubitus and limits mobilization, favouring alveolar derecruitment, atelectasis and posture lung injuries. Based on these findings, it seems difficult to recommend the ST for trauma patients at high-risk of developing delayed respiratory failure, when multiple emergent surgeries or ventral decubitus are planned, or when definitive stabilization is not expected in first days. EF has been shown to be in contrast reliable and relevant in these specific cases, with a possible maintaining during 3 weeks without compromising definitive osteosynthesis [26]. A prospective randomized study would be, however, necessary to confirm the superiority of EF on respiratory function.

Some limitations apply for the present series. First, as in other studies, there is a lack of statistical power, with a retrospective and monocenter design. Second, our study is somewhat comparable to a before/after study because of the change in DCO strategy during the study period (Fig. 2). The usual biases associated with this kind of design could, therefore, apply, particularly the associated changes in clinical practices. However, except during the admission period, trauma severity and the main admission characteristics were comparable between the two groups (Table 1). Third, timing until definitive fixation was slightly longer in the DCO-EF group ( $161 \mathrm{~h}$ versus $95 \mathrm{~h}, P=0.046$ ) (Table 2). The limits of ST that applied previously probably encouraged clinicians to anticipate performing definitive osteosynthesis. Furthermore, EF was the definitive treatment of the femoral fracture in our cohort for 6 patients. The maintaining of these patients may lead to analysis bias. We hypothesize that the timing may have been inappropriate or premature in some patients, promoting lung injuries and ARDS. This phenomenon could explain the differences observed between days 3 and 6 (Fig. 3a, b). Fourth, our high incidence of ARDS may seem surprising at first glance, because it was higher than in other series on DCO strategy $[5,6,27]$. This higher rate is certainly consequence of the definition of ARDS that we chose. The Berlin international definition highlights mild respiratory failure. The rate of moderate and severe ARDS $\left(\mathrm{PaO}_{2} / \mathrm{FiO}_{2}<200\right)$ was thus nearly similar to that in previous studies $[5,6,27]$. We assume that the detection of mild and moderate ARDS is one of the main strengths of our study. Previous work suggest when $\mathrm{PaO} 2 / \mathrm{FiO}_{2}$ ratio assessed under standardized ventilator settings, numerous patients with severe ARDS were reclassified as moderate, mild and nonARDS. Consequently, ARDS diagnosis based on $\mathrm{PaO}_{2} / \mathrm{FiO}_{2}$ ratio at ARDS onset may underestimated severity of ARDS [28]. Fifth, isolated contusions may not be properly considered as an ARDS. Indeed, lung contusion may be considered as a confusion factor and leads to false-positives for ARDS diagnosis. However, among the pathophysiological pathways involved in trauma-related ARDS, the activation of local and systemic inflammatory mechanisms, resulting from the activation of innate immunity, plays a key role. Nosological entities are numerous and potentiate themselves, such as contusion, fat embolism, alveolar collapse or pneumonia in later phase. All these entities may be superimposed and the distinction between each one of them is almost impossible. Sixth, we could not demonstrate a significantly higher risk 
of pseudarthrosis and inadequate consolidation with the ST device (Table 3). Our analysis certainly lacked statistical power regarding this. Consequently, no definitive conclusion can be drawn on these observations.

\section{Conclusion}

In this cohort of severe trauma patients with unilateral shaft fracture, we observed a higher incidence of ARDS in the DCO-ST group than in the DCO-EF group. Therefore, our data support that the prolonged use of ST might generate more lung injuries and ARDS. Consequently, the EF device would seem to be preferable in the setting of a DCO strategy for trauma patients at risk of delayed respiratory failure. A prospective randomized study is necessary to confirm this.

\section{Compliance with ethical standards}

Conflict of interest All the authors declare that they do not have any financial and personal relationships with people or organization that can inappropriately influence the work. There is no professional or other personal interest of any nature or kind in any product, service and/or company that could be construed as influencing the position of the authors.

\section{References}

1. Scalea TM. Optimal timing of fracture fixation: have we learned anything in the past 20 years? J Trauma. 2008;65(2):253-60.

2. Gray AC, White TO, Clutton E, Christie J, Hawes BD, Robinson CM (2009). The stress response to bilateral femoral fractures: a comparison of primary intramedullary nailing and external fixation. J Orthop Trauma 23(2), 90-97 (discussion 98).

3. Buzdon MM, Napolitano LM, Shi HJ, Ceresoli DM, Rauniya R, Bass BL. Femur fracture induces site-specific changes in T-cell immunity. J Surg Res. 1999;82(2):201-8.

4. Lefaivre KA, Starr AJ, Stahel PF, Elliott AC, Smith WR (2010) Prediction of pulmonary morbidity and mortality in patients with femur fracture. J Trauma 69(6), 1527-35 (discussion 1535).

5. Pape HC, Hildebrand F, Pertschy S, Zelle B, Garapati R, Grimme $\mathrm{K}$ et al. (2002) Changes in the management of femoral shaft fractures in polytrauma patients: from early total care to damage control orthopedic surgery. J Trauma 53(3), 452-61 (discussion 461).

6. Pape HC, Giannoudis PV, Krettek C, Trentz O. Timing of fixation of major fractures in blunt polytrauma: role of conventional indicators in clinical decision making. J Orthop Trauma. 2005;19(8):551-62.

7. Steinhausen E, Lefering R, Tjardes T, Neugebauer EA, Bouillon B, Rixen D; Committee on Emergency Medicine, Intensive and Trauma Care (Sektion NIS) of the German Society for Trauma Surgery (DGU). A risk-adapted approach is beneficial in the management of bilateral femoral shaft fractures in multiple trauma patients: an analysis based on the trauma registry of the German Trauma Society. J Trauma Acute Care Surg 2014;76(5):1288-93.

8. Taeger G, Ruchholtz S, Waydhas C, Lewan U, Schmidt B, Nast-Kolb D. Damage control orthopaedics in patients with multiple injuries is effective, time saving, and safe. J Trauma. 2005;59:408-15.
9. Scalea TM, Boswell SA, Scott JD, Mitchell KA, Kramer ME, Pollak AN (2000) External fixation as a bridge to intramedullary nailing for patients with multiple injuries and with femur fractures: damage control orthopedics. J Trauma 48(4):613-21 (discussion 621)

10. O'Toole RV, O'Brien M, Scalea TM, Habashi N, Pollak AN, Turen $\mathrm{CH}$. Resuscitation before stabilization of femoral fractures limits acute respiratory distress syndrome in patients with multiple traumatic injuries despite low use of damage control orthopedics. J Trauma. 2009;67(5):1013-21.

11. Scannell BP, Waldrop NE, Sasser HC, Sing RF, Bosse MJ. Skeletal traction versus external fixation in the initial temporization of femoral shaft fractures in severely injured patients. J Trauma. 2010;68(3):633-40.

12. Hamada SR, Gauss T, Duchateau FX, Truchot J, Harrois A, Raux $\mathrm{M}$, et al. Evaluation of the performance of French physicianstaffed emergency medical service in the triage of major trauma patients. J Trauma Acute Care Surg. 2014;76(6):1476-83.

13. Baker SP, O'Neill B, Haddon W, Long WB. The injury severity score: a method for describing patients with multiple injuries and evaluating emergency care. J Trauma. 1974;14(3):187-96.

14. Daurat A, Millet I, Roustan JP, Maury C, Taourel P, Jaber S, et al. Thoracic Trauma Severity score on admission allows to determine the risk of delayed ARDS in trauma patients with pulmonary contusion. Injury. 2016;47(1):147-53.

15. Vincent JL, Moreno R, Takala J, Willatts S, De Mendonça A, Bruining $\mathrm{H}$ et al. (1996) The SOFA (sepsis-related organ failure assessment) score to describe organ dysfunction/failure. On behalf of the working group on sepsis-related problems of the European Society of Intensive Care Medicine. Intensive Care Med 22(7), 707-10.

16. Wagstaff TA, Soni N. Performance of six types of oxygen delivery devices at varying respiratory rates. Anaesthesia. 2007;62(5):492-503.

17. The ARDS Definition Task Force, Ranieri VM, Rubenfeld GD, Thompson BT, Ferguson ND, Caldwell E, Fan E, Camporota L, et al. Acute respiratory distress syndrome: the Berlin definition. JAMA 2012;307(23):2526-33.

18. Seibel R, Laduca J, Hassett JM, Babikian G, Mills B, Border DO, et al. Blunt multiple trauma (ISS 36), femur traction, and the pulmonary failure-septic state. Ann Surg. 1985;202(3):283-95.

19. Bone LB, Johnson KD, Weigelt J, Scheinberg R. Early versus delayed stabilization of femoral fractures: a prospective randomized study. J Bone Joint Surg Am. 1989;71(3):336-40.

20. Pape HC, Schmidt RE, Rice J, van Griensven M, das Gupta R, Krettek C, et al. Biochemical changes after trauma and skeletal surgery of the lower extremity: quantification of the operative burden. Crit Care Med. 2000;28(10):3441-8.

21. Giannoudis PV, Smith RM, Bellamy MC, Morrison JF, Dickson RA, Guillou PJ. Stimulation of the inflammatory system by reamed and unreamed nailing of femoral fractures: an analysis of the second hit. J Bone Joint Surg Br. 1999;81(2):356-61.

22. Lasanianos NG, Kanakaris NK, Dimitriou R, Pape HC, Giannoudis PV. Second hit phenomenon: existing evidence of clinical implications. Injury. 2011;42(7):617-29.

23. Pape HC, Giannoudis P, Krettek C. The timing of fracture treatment in polytrauma patients: relevance of damage control orthopedic surgery. Am J Surg. 2002;183(6):622-9.

24. Scalea TM, Boswell SA, Scott JD, Mitchell KA, Kramer ME, Pollak AN (2000) External fixation as a bridge to intramedullary nailing for patients with multiple injuries and with femur fractures: damage control orthopedics. J Trauma 48(4), 613-21 (discussion 621).

25. Pape HC, Rixen D, Morley J, Husebye EE, Mueller M, Dumont $C$, et al. EPOFF Study Group. Impact of the method of initial stabilization for femoral shaft fractures in patients with multiple 
injuries at risk for complications (borderline patients). Ann Surg 2007;246(3):491-9 (discussion 499).

26. Harwood PJ, Giannoudis PV, Van Griensven M, Krettek C, Pape HC (2005) Alterations in the systemic inflammatory response after early total care and damage control procedures for femoral shaft fracture in severely injured patients. J Trauma 58(3), 446-52 (discussion 452).
27. Caba-Doussoux P, Leon-Baltasar JL, Garcia-Fuentes C, ResinesErasun C. Damage control orthopaedics in severe polytrauma with femur fracture. Injury. 2012;43(suppl 2):S42-S4646.

28. Villar J, Blanco J, del Campo R, Andaluz-Ojeda D, DíazDomínguez FJ, Muriel A, et al. Assessment of $\mathrm{PaO}_{2} / \mathrm{FiO}_{2}$ for stratification of patients with moderate and severe acute respiratory distress syndrome. BMJ Open. 2015;5(3):e006812. 